\title{
How to Design the Registration and Login Function of APP
}

\author{
Zhengyou Wang ${ }^{1}$, Yan Sun ${ }^{2}$ \\ ${ }^{1}$ Department of Film and Television Arts, Shanghai Publishing and Printing College, Shanghai, China \\ ${ }^{2}$ College of Communication and Art Design, University of Shanghai for Science and Technology, Shanghai, China \\ Email: wangzy59@163.com, sunyan1542@163.com
}

How to cite this paper: Wang, Z.Y. and Sun, Y. (2018) How to Design the Registration and Login Function of APP. Journal of Software Engineering and Applications, 11, 223-234.

https://doi.org/10.4236/jsea.2018.115014

Received: May 10, 2018

Accepted: May 22, 2018

Published: May 25, 2018

Copyright () 2018 by authors and Scientific Research Publishing Inc. This work is licensed under the Creative Commons Attribution International License (CC BY 4.0).

http://creativecommons.org/licenses/by/4.0/

\begin{abstract}
The humanization design, friendly user experience shall be very important for mobile phone interface. The mobile interface design shall be such an important direction for the research of human-computer interaction techniques. The main purpose of this paper is to discuss the design principles, methods and design process for APP registration and login function. In this paper, it has expounded how to judge the APP needs registration and login functions, and then gave the brief elaboration on several commonly-used registration and login methods. Finally, it discusses how to design APP registration and login functions accordingly.
\end{abstract}

\section{Keywords}

UI, Interface Design, Human-Computer Interaction

\section{Introduction}

The demand for registration and login may be caused by the need to establish user system, or the need to collect user information, or the need for user operation etc. To realize "registration" and "login" function shall have the vital effect for business, and it can also play the important influence on so many business functions, and such work may need the careful and cautious design accordingly.

The key point for whether an APP needs registration and login depends on the product form of such APP [1].

If user's registration and login cannot bring any further value to user's demand, product function and business model itself, it shall be unnecessary to design such function accordingly, for example, those products in practical tools category like calculator, flashlight etc.

It may be for the need to establish the user system, or the need to collect user 
information, or the need for user operation etc., and following APP in certain types may need the design of registration and login. Some strong-ties social software products, like WeChat, facebook etc. concerned so many user's treasure security, like Alipay; some products focused on user's attention to information even more, like e-mail etc.; in addition, some other APPs in entertainment, game categories, all need the support of account system; of course, they also need the function of registration and login.

\section{The Value of Registration and Login Functions}

First of all, we'd analyze where shall be the value of registration and login functions. The analysis can be divided into three levels of user, product function and business:

\subsection{User Level}

From the perspective of the user, the registration and login functions shall be: the user needs to get an independent ID in such product, to represent his independent existence accordingly. The user may need to record his behavior trace, such as using progress, likes, the favorite content etc. The user also needs to establish connection with other users, access the real information or detailed information of other users accordingly. The user may also needs the product to get to know himself in details, so as to provide better service to him.

\subsection{Product Function Level}

From the perspective of product function, the registration and login function shall be: to bring convenience for data synchronization cross different equipment, also bring convenience for switching different accounts in the same equipment and maintaining the previous status of the account. The products with social function must register the account to compile its own UGC content, meanwhile bring the convenience for identification by other users. Those products with transaction function can record the transaction information for personal accounts meanwhile improve the security of transaction. The products with paid charges inside application and value-added function for VIP membership can ensure its user's interests and rights through registration and login.

\subsection{Business Level}

From the perspective of business, the registration and login function shall be: to gather those user information that are very important for the core business [2], such as the information of mobile phone number, real name etc. After registration and login, it can bring convenience for gathering data that can promote operation, so as to guide the direction for product operation accordingly. User incentives: through registration to get the mobile phone number and e-mail address, some products can attract the users through sending short message or discount coupon, to improve user activity degree accordingly. Consumption 
transformation: after getting mobile phone number, some products will market their paid service to customer through the method of telephone marketing.

To sum up, before designing registration and login functions, the designer should figure out how many above-mentioned contents its product target should have, what about the importance of account registration function, and which core function shall have relationship with it accordingly.

\section{Classification of Registration and Login Methods}

\subsection{Registration Methods}

The registration methods are diversified, mainly the 4 major types: mobile phone registration, e-mail registration, registration with customized user name, registration by adding invitation code.

\subsection{Login Methods}

The login methods are mainly following types: account login, such as mobile phone, e-mail box; third party login such as We Chat, QQ, Weibo; biological nature login such as fingerprint, vocal print, human face, iris; one-key quick login such as Baidu's account linkage login, as per shown in Figure 1.

\subsection{The Merit and Demerit of Several Registration and Login Methods}

Registration and login were unified, the user can log in directly through mobile phone verification code. Such method firstly does not need complex registration procedure and also do not require the user to remember its password. Secondly it can guarantee account security and identity authentication of the account, greatly reduce the possibility of malicious registration and improve user quality. But its demerit was also existed, because there's no password, for the platform with site at PC end, the users must take their own mobile phone for registration, and this shall not be so convenient.

Registration and login were separated, the user firstly needs to register and set the password accordingly. Through such method it can gain user's password information while making login through multiple platforms it shall bring better user experience. Of course such method did also have the obvious demerit, the user may easily feel bored about such complex procedure and the user's interest shall be consumed during the registration process. And this may also cause the increased churn rate greatly.

One-key login may need higher authorization; APP backstage will send a short message to serve and arrange automatic login after gaining the authorization; the password can be changed independently in personal configuration.

In comparison with self-owned account system, the third party account system shall be quite convenient and easy to use; in China there are three major social platform authorization login in We Chat, Weibo and QQ [3]; abroad China there are also the common-used Facebook, Twitter and Google. First of all, in 


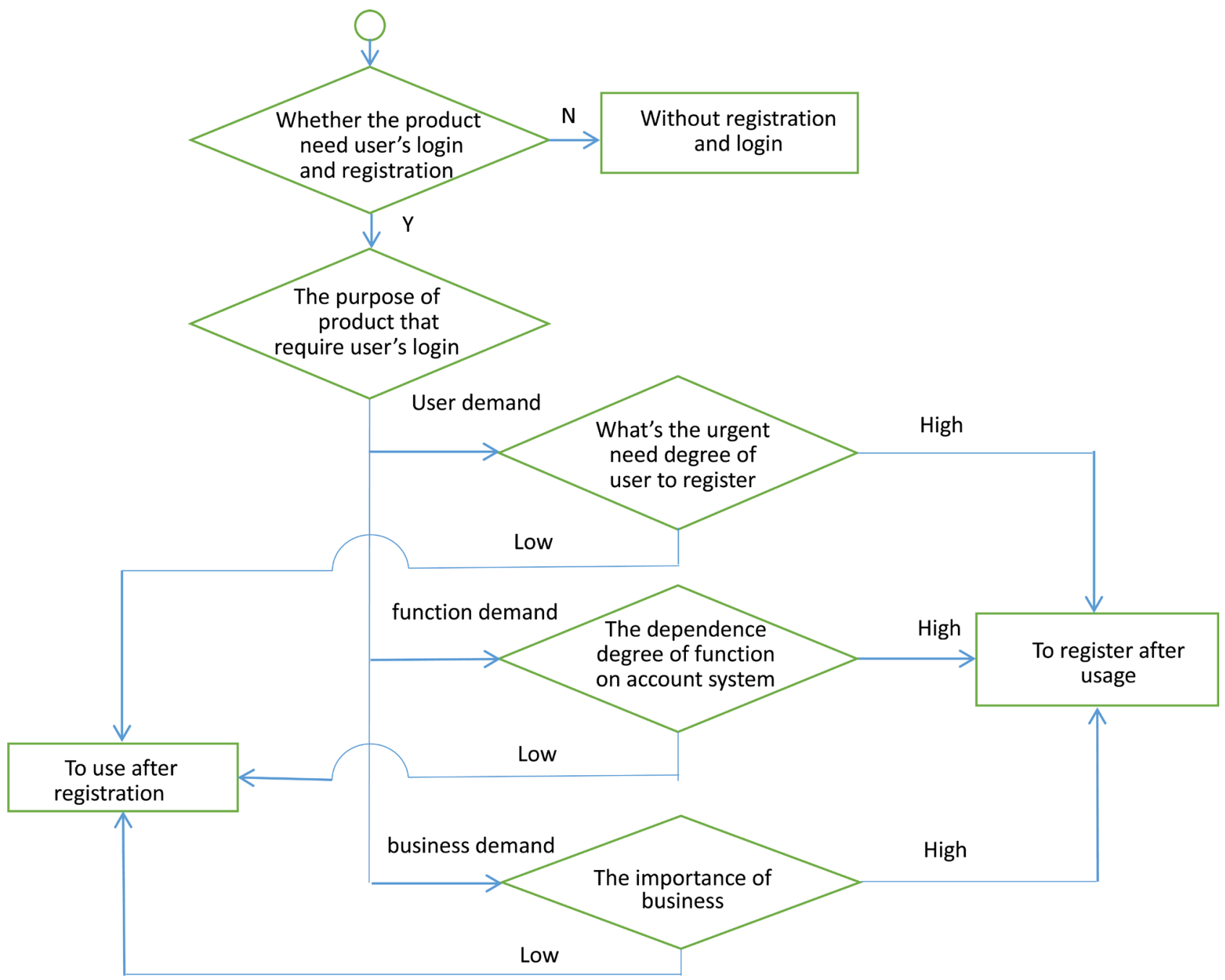

Figure 1. Design thought of registration and login.

order to save user's registration time; it can be logged in just through only two simple clicks. Secondly, those users who have registered through third party platform have also passed through the verification with mobile phone and e-mail box; it's secured and reliable. Meanwhile, by introducing the third party account, it also push the problem of account security to the third party platform. Finally, by utilizing the third party account login, it can access the information of third party platform under convenient conditions, such as friends info, basic information etc. Such method shall be suitable for the situation that the user was not familiar with such product; then the user can experience such product without registering a new account. And the final purpose of such product shall also be to precipitate users and achieve more user information.

At last, there is still another e-mail box registration method in the separated registration and login methods. In comparison with mobile phone number registration, the merit of e-mail box registration was that the e-mail box address can be permanently preserved, without the trouble caused by changed mobile phone number. But it also has had may demerits, first of all, for domestic users, their 
use frequency was not higher as the foreigners; the importance of e-mail box was even lower than that of QQ, We Chat and mobile phone; there were so many users have no e-mail box; and they were also not willing to apply for personal e-mail box just because they'd register an account, so that they may have relative weak demand on e-mail box registration. Secondly, it did also have poor experience to register through e-mail box at mobile terminal, because the e-mail box verification needs to quit the APP and receive the e-mail, whatever it logged in e-mail client or opened the browser to enter into e-mail home page, such operation shall both raise the complexity of such procedure and reduce user's experience. But with the consideration of that the APP user group shall not only contain domestic users, the e-mail box registration was also retained for the consideration of foreigner's use habit. Some other business APP locked their user group as the professionals, these people shall have the habit to use e-mail box. Some study APP has considered that the student group may have bigger possibility to change their account. Some other users did not want their mobile phone number being exposed; they shall be the user groups that are unwilling to register with mobile phone number.

\section{The Design thought of Registration and Login Methods}

During the product design process, there shall not be the standard answer on how to select the registration and login method and design the procedure of registration and login according to the different stage of product, different product target, different group positioning. Followed is such an overall analysis thought for registration and login method [4].

\subsection{Whether the Registration and Login Function was Needed}

To analyze necessity for product requiring user registration and login. If user registration and login cannot bring any value to the user, product function, business model itself; it's really unnecessary to design such function, as per shown in Figure 1. For example some products in practical tools category: calculator, flashlight, weather forecast, Android cleaner master, vocal assistant etc.

\subsection{The Purpose for Designing User Registration and Login}

What kind of value the user registration and login function can bring to the product, then it can result in the judgment on whether such APP should be used after registration or registered after usage, as per shown in Figure 2. Such problem can be analyzed through the three levels of user, function and business.

1) user's perspective

To analyze the urgent need degree of user to register the product. Once the user installed and used an APP at first time, he/ she may have three different psychologies:

the user has strong willing to use the product

The one situation is that the product function shall be more valuable for user, 


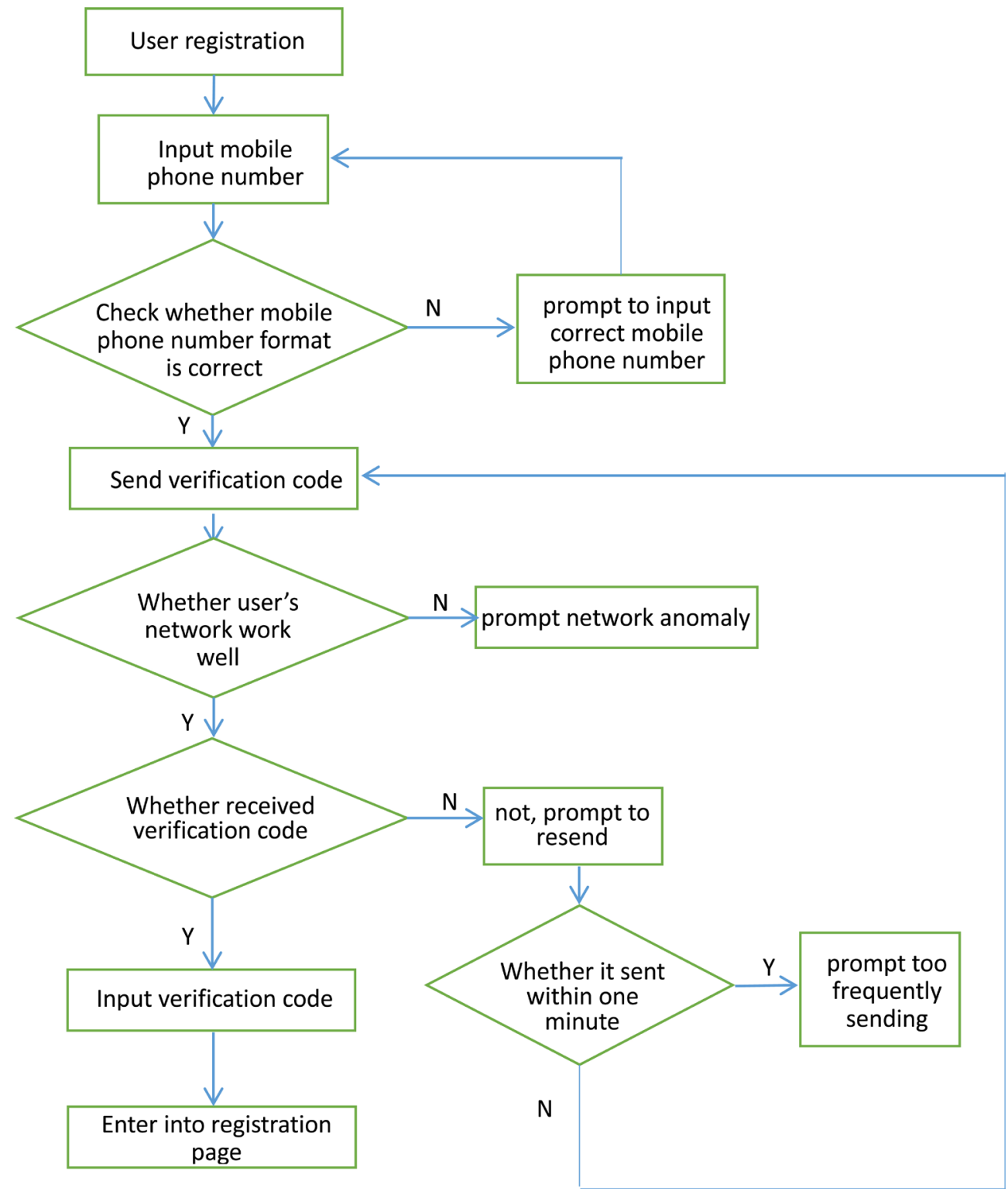

Figure 2. The procedure of inputting mobile phone number.

the user is also willing to spend time for registration and use, for example some hand games. Another situation is that product function was quite strange and fresh, the user may hope to have a try sooner as possible, such as FaceU. For those products the user may have strong will to use, it can certainly let the user using it after registration.

the user wants to have a trail first.

The user hops to have a trail use, if it's good enough he/she may start to register. This shall be the same as the thought of experiencing consumption, buying after trail. For most users must hold the attitude to have a try to use at first time, in order to leave the best primary impression on the users, the procedure of registration after use shall be necessary. Currently most products did provide such method. If your APP cannot make the user creating strong will to use, the best 
choice shall be let the user using it, once the users thought it's good enough then ask them to register accordingly.

the user does not feel it's necessary to register.

The user feels it's not necessary to register, but the product function can be used occasionally. Some APP with lower usage frequency like ring tones, wall paper software, and some other software in tools + social category, for the users the registration function cannot bring any value to them, it was suggested that those APP use the method of registration after use.

\section{2) functional demand}

By thinking from the perspective of function, the one situation is that the core function of product did depend on user system too much; it can be used after registration. For example those social software like QQ. We Chat, Momo etc., without login it cannot use the normal function. Another situation is that some product functions may need user login, but it shall not impact other functions, it can require to $\log$ in once such function was needed. For example, the e-commerce APP can be viewed first, then to log in while adding some items into shopping trolley or making the order.

3) business demand

By judging from perspective of business, if the registration information was very important for product's core business, it can consider of registration-first model, or the common measure shall be that the first page for APP entrance was the registration menu but the button of "visitor's entrance" was left at the corner. Of course if it just for the purpose of getting user's mobile phone number, it shall not be necessary to use such way through registration [5]. It can guide the user to input information or register in the product function page. For example the small testing function of "help you choose your car" in driving test treasury book.

\subsection{The Scenario of Registration and Login Demand}

To analyze how's about each scenario of registration and login demand, according to the analysis on the scenario to solve the problem on how to get the registration and login method most suitable for this APP. The thought was still made through the three levels of user, function and business.

1) functional demand

Based on the perspective of function, a reasonable account system shall be needed for such a function with the demand of registration and login, and it's really not important to choose which kind of registration method at all. So that the influence of functional demand on the choice of registration and login method can be ignored accordingly.

2) user's demand

The three registration and login methods shall have the obvious merits and demerits for user, users' different characteristic of personality, living habit will also influence their choice: for example, some users hated to disclose their mo- 
bile phone number, some users have no personal e-mail, some users did not install Weibo etc. In addition, the same user shall also have different choice under different situations: for example, to use in metro or bus, dining, while watching, boring, recommended by friend nearby etc. With the consideration of those diversified scenario demand, the most reasonable measure should be to provide multiple registration and login methods for the user, better with all the methods of mobile phone number, e-mail box and third party registration, let the users making their own choice accordingly.

To make further analysis, if the target user group of APP can be locked, it can refine the user scenario highlighting its characteristics and can also add or reduce registration and login methods according to the characteristic of its users. For example, without consideration of overseas user group, the user did also have no habit to use e-mail; then the e-mail registration method could be ignored accordingly. For example, most of the users were the college students, they may usually change mobile phone number, or some of them may have the possibility to study abroad, then e-mail registration shall be necessary.

\section{3) business demand}

By analyzing the specific business demand scenario, it can get different choices. For some business models, the information of user's mobile phone number should be very important, it may consider to only keep one method of mobile phone registration and do not give further more choices to the user. For some products, it may consider of the factors of developing cost and schedule, to choose the most commonly used mobile phone number registration method. For some other products, the mobile phone number shall not be the necessary information; it can choose the third party account login method directly.

\subsection{The Procedure Design of Login System}

Login registration usually contains three modules as registration, login and forget password, to take mobile phone number as example, the APP registration system procedure includes three pages such as inputting mobile phone number, registration page and filling in personal information, each page shall contain the normal status, inputting status and feedback status [6], the routine procedure was shown in Figure 2 and Figure 3.

Once the user completed the mobile phone number input page and registration page, it then enter into filling in personal information page, in this page whatever the user has inputted information, the registration will be finished accordingly.

By taking mobile phone number login module as example, the APP login system procedure includes one page of user login, the page contains the normal status, inputting status and feedback status, its routine procedure was shown in Figure 4.

By taking the module of forget password for mobile phone number login as example, the APP forget password system procedure contains two pages of in- 
putting mobile phone number, revising page, each page includes the normal status, inputting status and feedback status, its routine procedure was shown in Figure 5 and Figure 6.

\subsection{Improvement Suggestion}

1) ask the user to fill in less information at registration page

In the new user registration page, do not ask excessive information, try to let
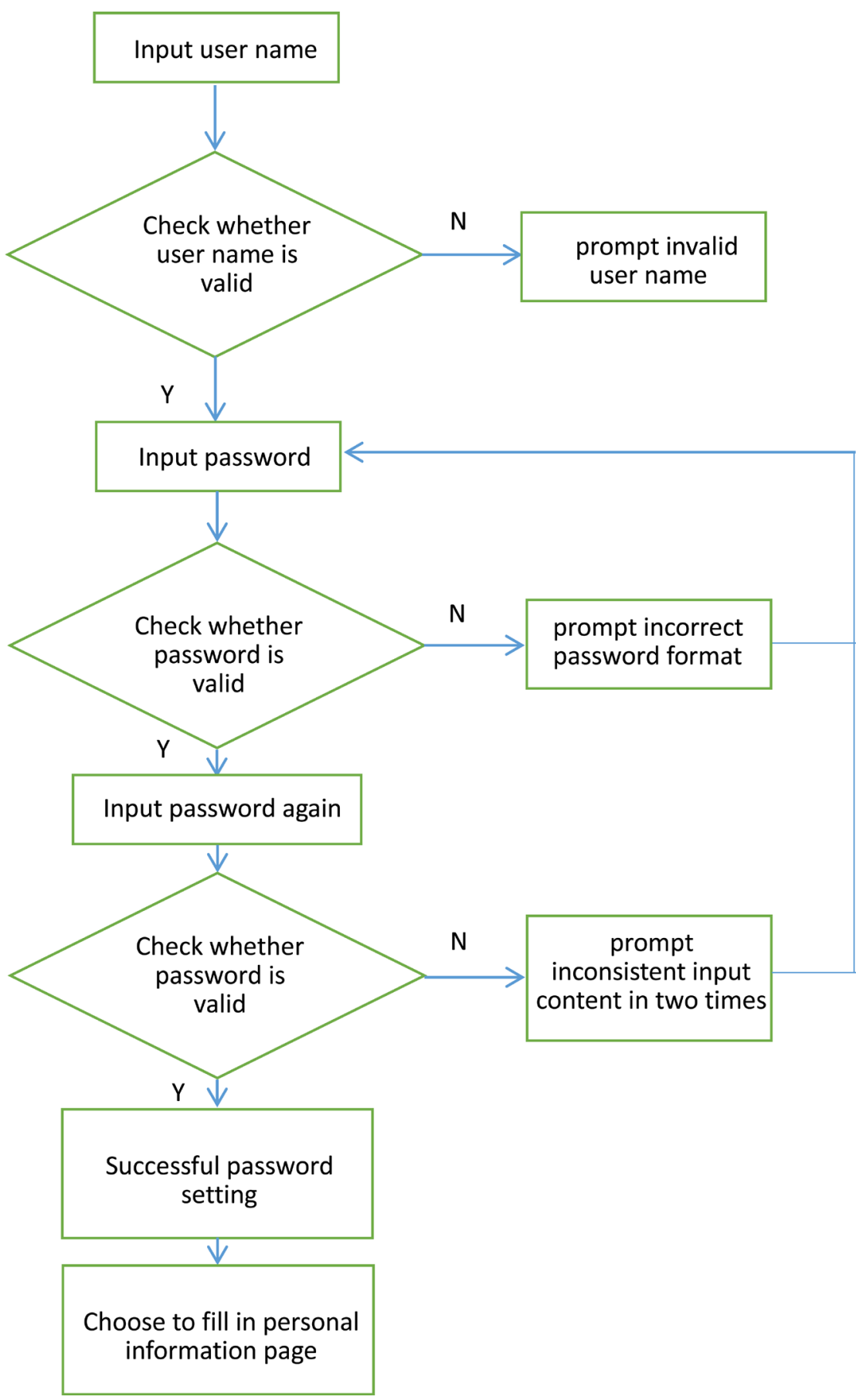

Figure 3. The procedure of user registration. 


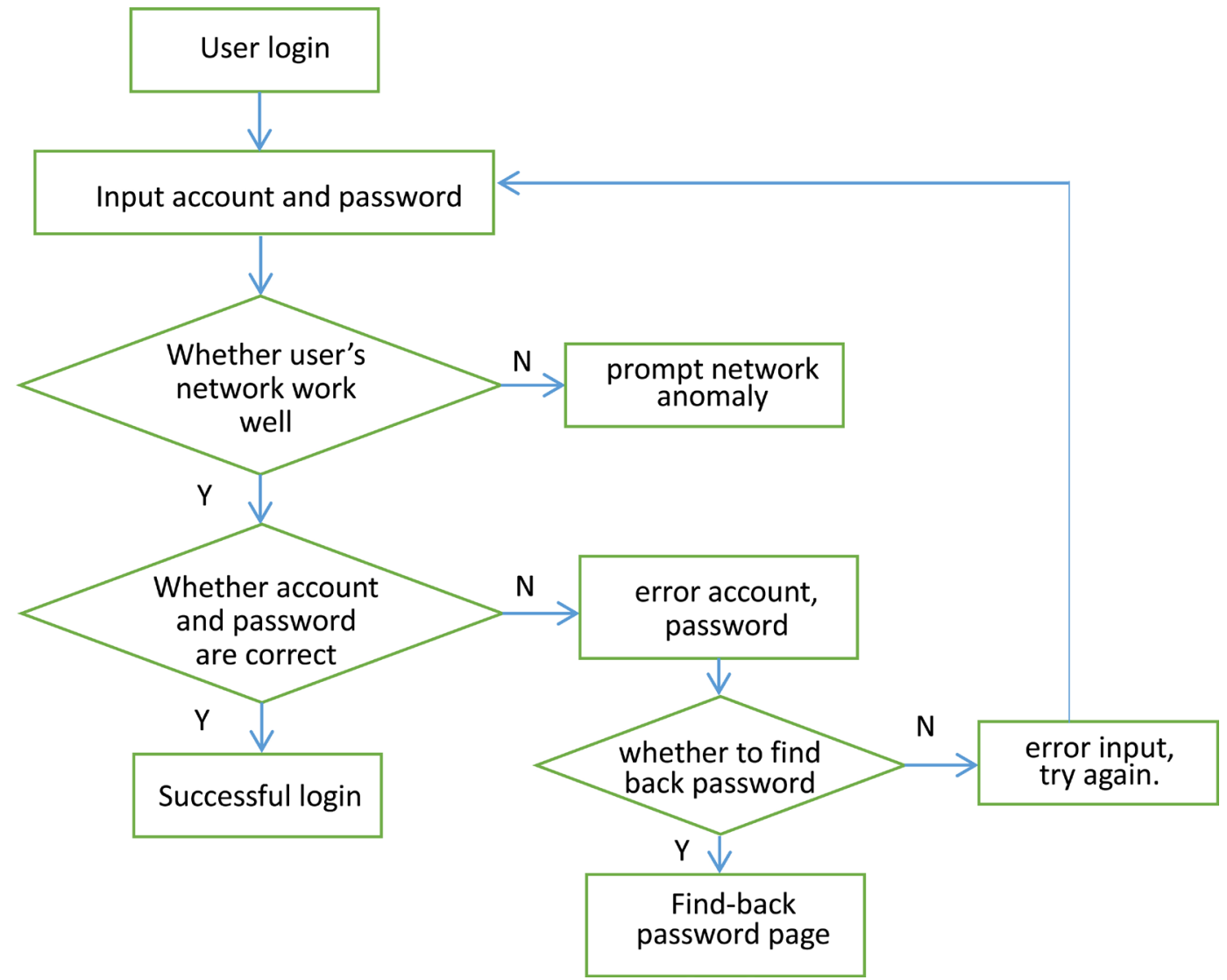

Figure 4. The procedure of user login.

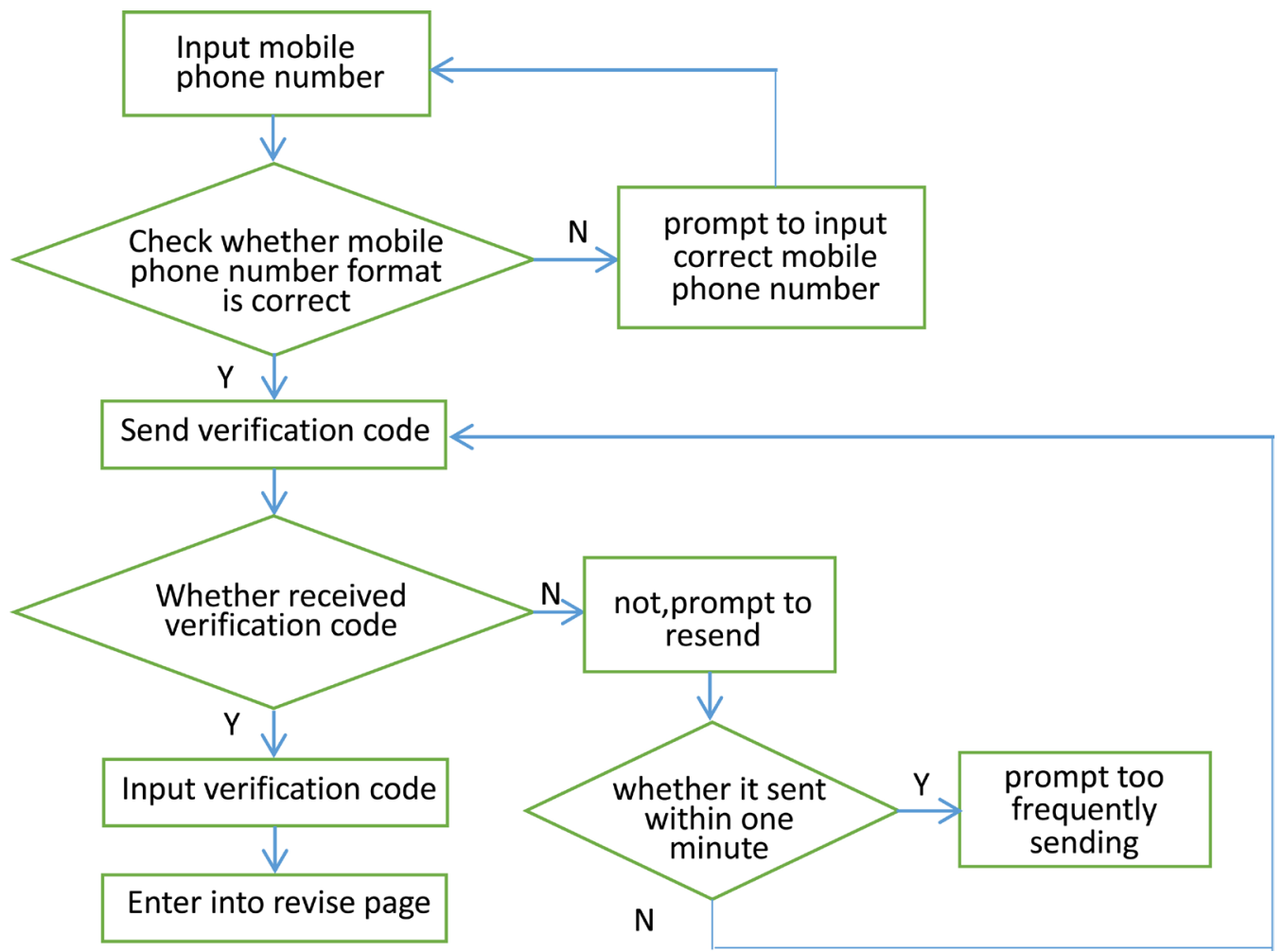

Figure 5. The procedure of forgetting password. 


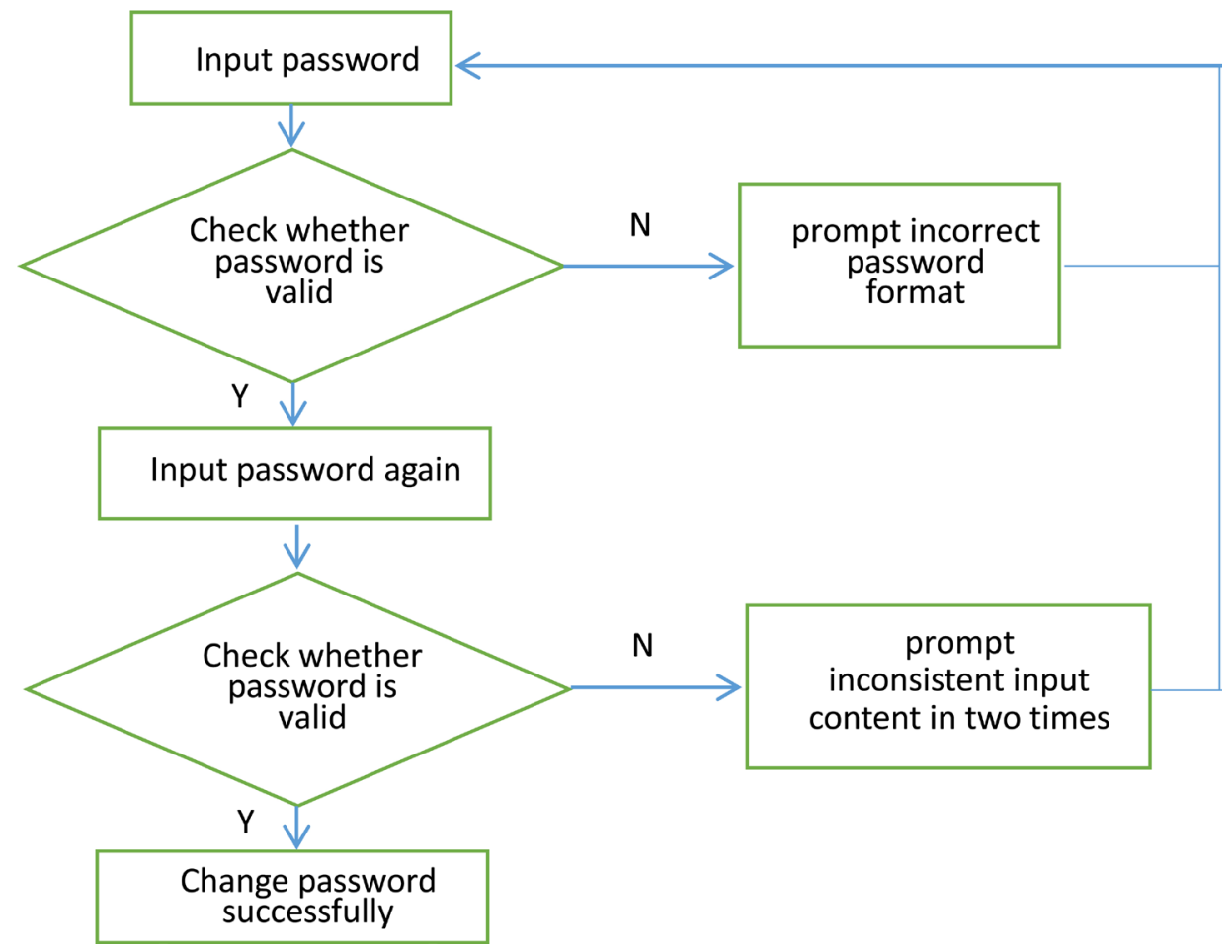

Figure 6. The procedure of changing password.

the user completing registration as soon as possible. The less input boxes you've set in the sheet, the lower possibility the user may choose not to fill in it. Before designing registration page, the designer must think clearly what kind of user information you really need. In addition, it should also pay attention to following points while starting design:

Do not ask the user to input password repeatedly

Try to reduce the unnecessary required fields as much as possible

2) to simplify the process of password resetting

The user may forget the password, thus it's particularly important to find back the password. Try to simplify the procedure of finding back password can improve user's stickiness. Put the link of "find back password" at the login/ registration page and let it being displayed constantly, rather than to pop up such link while the user inputting password incorrectly.

\section{What We Can Do More?}

During above process design, there shall be so many details that need to be focused and set according to the demand. For example, whether it needs to remember password? Or automatic login in within two weeks? How to prompt the user for a password and check its strength? How to design the better error prompt for registration page? If there is a user image in registration page, whether it needs to lock the image proportion? Whether it provides online tailoring? Whether it needs to set the default image for the user?

To sum up, during software development process, it should pay attention to 
the demands in three aspects of customer, software function and business, with the premise of ensuring complete procedure design, to guarantee the perfect design of user experience, functional logic and security as much as possible.

\section{References}

[1] Su, W.Z. (2017) The User Experience Research in UI Design. Wireless Technology, No. 2.

[2] Rao, W.M. and Lin, D.G. (2016) Just Talk about Smart Phone App UI Design Techniques in the Development. Electronic Test, No. 13.

[3] Zhang, P. (2016) Writing the Android Mobile Platform UI Design Research. Nanjing Normal University, Nanjing.

[4] Tu, X.D. (2010) Introduction to the UI Design. Computer Knowledge and Technology, No. 7.

[5] Hu, X.D. (2014) The Design and Implementation of a Music Player Based on Android Platform. Jilin University, Changchun.

[6] Tian, L.G. and Yan, H. (2014) Smart Phone APP Interface Design Study. Western Radio and Television, No. 21. 